\title{
Laparoscopic spleen-preserving distal pancreatectomy with intraoperative vascular repair
}

\author{
Xin Wang $\cdot$ Yongbin Li $\cdot$ Bing Peng
}

Received: 19 January 2013/Accepted: 14 June 2013/Published online: 1 January 2014

(C) Springer Science+Business Media New York 2013

\begin{abstract}
Laparoscopic vascular repair is technically challenging in pancreatic surgeries. We present a case of a 42-year-old woman who was initially admitted to our hospital because of upper abdominal pain. Preoperative abdominal enhanced CT scan revealed a cystic mass near the spleen that was $4.7 \times 4.3 \times 3(\mathrm{~cm})$ in dimension. Therefore, we performed laparoscopic spleen-preserving distal pancreatectomy. In this procedure, we repaired spleen-related vessels with laparoscopic suture due to the adhesion between the vessels and the tumor. Finally, the spleen was preserved with good blood supply. This procedure took $200 \mathrm{~min}$ and the estimated blood loss was
\end{abstract}

$360 \mathrm{ml}$. The postoperative abdominal Doppler ultrasound carried out 6 days after surgery indicated blood supply to the spleen was good and there was no thrombosis. The patient was discharged without any complications on postoperative day 7 . The pathological diagnosis was pancreatic mucinous cystadenoma.

\section{Keyword Pancreatic}

Disclosures Xin Wang, Yongbin Li, and Bing Peng have no conflicts of interest or financial ties to disclose.
Presented at the Society of American Gastrointestinal and Endoscopic Surgeons (SAGES) 2013 Annual Meeting, Baltimore, MD, USA, 17-20 April 2013.

Electronic supplementary material The online version of this article (doi:10.1007/s00464-013-3328-2) contains supplementary material, which is available to authorized users.

X. Wang $\cdot$ Y. Li · B. Peng $(\bowtie)$

Department of Hepatopancreatobiliary Surgery, West China

Hospital, Sichuan University, Chengdu 610041, China

e-mail: hxwangxin2012@hotmail.com 